\title{
Maestras y pioneras: condiciones para el acceso de la mujer a la educación superior en Colombia en la primera mitad del siglo XX
}

Teachers and Pioneers: conditions for women's access to higher education in Colombia in the first half of the 20th century

\section{REsUMEN}

El objetivo de este texto es analizar condiciones culturales, políticas y económicas que permitieron que desde inicios del siglo XX las mujeres en Colombia se abrieran paso en la formación universitaria y en la administración pública. Para esto, se hizo una revisión de prensa y se analizaron fuentes secundarias para identificar casos particulares de mujeres que a principios del siglo XX tuvieron acceso a la educación superior y cuya formación contribuyó al acceso de otras mujeres a la formación universitaria. A partir de este análisis, se encontró que el acceso de las mujeres a la educación fue resultado de condiciones interdependientes y no de situaciones particulares aisladas en las que resultó fundamental la formación inicial que como maestras recibieron dichas mujeres.

Palabras clave: mujeres, maestras, educación superior, pioneras, interdependencias, participación femenina.
Marinella Lozano-Cruz

Magíster en Historia, estudiante

Doctorado en Historia

Universidad Nacional de

Colombia, sede Medellín.

\mlozanoc@unal.edu.co

(1) ORCID: 0000-0002-9469-8520

$\checkmark$ Google Scholar 


\begin{abstract}
The objective of this text is to analyze cultural, political and economic conditions that allowed women in Colombia to make their way into university education and public administration since the beginning of the 20th century. For this, a press review was made and secondary sources were analyzed in which particular cases of women who had access to higher education and whose training contributed to the access of other women to university training were identified at the beginning of the 20th century. From this analysis, it was found that women's access to education was the result of interdependent conditions and not of particular isolated situations in which the initial training that such women received as teachers was essential.
\end{abstract}

Keywords: women, teachers, higher education, pioneers, interdependencies, female participation.

\title{
Presentación
}

\author{
"Un pedacito de gloria tenemos ya en las manos \\ nosotras las más oscuras. Las más afortunadas han \\ dado un impulso vigoroso a la lenta rueda de nuestro \\ avance intelectual y otras vamos tras de ellas, anhelosas \\ y felices, a aumentar el número de las labradoras de \\ nuestra propia y común redención" (Camacho, 1926b).
}

n la primera mitad del siglo XX se dieron en Colombia trans-
formaciones que permitieron el acceso de las mujeres a la
educación superior. En este sentido, es claro que, aunque en primera instancia el oficio de maestras se convirtió para las mujeres en una alternativa casi exclusiva de educación, hubo mujeres que desde su formación de maestras abrieron el camino para que muchas otras pudieran optar por formarse en otras profesiones distintas a la docencia. De esta manera, resultan importantes las gestiones que desde las instituciones de enseñanza femenina se dieron y que hacen parte de los esfuerzos por abrirles paso a las mujeres en el escenario de la formación universitaria. Así, por ejemplo, es notable el papel del Instituto Pedagógico Nacional para Señoritas, puesto que representó un lugar importante para el desarrollo de la educación femenina en el país. Allí se gestaron algunas de las condiciones que les permitieron a las mujeres acceder a un título que posteriormente, les brindó la posibilidad de ocupar un espacio en la política educativa del país y en el fortalecimiento de la formación superior de otras mujeres. 
En la historia de la educación colombiana, son muchos los nombres de mujeres que representan un aporte en ese camino que se forjó. En consecuencia, el ingreso de la mujer en este campo no fue el resultado de experiencias personales extraordinarias, sino que hace parte de una serie de interdependencias que contribuyeron a ello. Lo que aquí se espera entonces, es identificar las condiciones en las que se dio el acceso de las mujeres a la educación superior; analizar dichas condiciones en el marco de un escenario social, político y económico más amplio, que permita entender la relación entre las primeras mujeres maestras y la ampliación del ingreso de las mujeres a la educación superior.

De esta forma, se espera realizar un aporte a los estudios sobre la historia de la educación en Colombia, no solo desde una perspectiva de género -aun cuando la historia que en este análisis se aborde sea la historia de maestras que se consideran aquí pioneras-, sino desde una perspectiva mucho más amplia en la que la historia de las mujeres no ocupe un lugar subordinado dentro del campo; es decir, que desde esta perspectiva, se pretende que dicha historia sea reconocida como parte de la historia de la educación colombiana y no solo como la historia de un grupo particular de mujeres, ya que el aporte que dichas mujeres realizaron no puede reducirse a un asunto de género.

\section{Pioneras: inicios de la formación universitaria femenina}

Para iniciar, es pertinente señalar que el lugar común en el análisis de la historia de la educación en Colombia, es el señalamiento de la escasa formación que recibían las mujeres y su segregación respecto a la educación recibida por los hombres ${ }^{1}$. Sin embargo, las tasas de alfabetización registradas para 1918, por ejemplo, muestran porcentajes similares entre hombres y mujeres, de manera que la situación de analfabetismo no era una simple exclusión de las mujeres, sino una situación generalizada en la población (Helg, 1987, p. 36). En este sentido, si bien es cierto que había limitaciones frente a la posibilidad de las mujeres para educarse, es necesario reconocer, como lo señala Helg por ejemplo, que para 1931, de los 3.849 maestros que había en las zonas rurales en Colombia, el $90 \%$ eran mujeres; empero, el 90 \% de ellas no tenía formación superior.

En relación con lo anterior, Helg (1987) afirma que en su mayoría estas maestras provenían de las clases medias de pueblos y ciudades 
pequeñas; no contaban sino con cuatro o seis años de escolaridad y en algunos casos, habían recibido formación durante unos años en colegios locales privados. De acuerdo con esta autora, "la enseñanza rural estaba pues confiada a mujeres sin diploma y solteras" (p. 51). Asimismo, Helg menciona que algunas de ellas poseían títulos de maestras que no eran reconocidos por el Estado. De esta manera, "el oficio de maestra de escuela representaba, para las jóvenes de su clase, la única posibilidad de no ser costurera ni entrar a un convento" (p. 53).

Una de las jóvenes que se formó como maestra y que hizo parte de las transformaciones que en el acceso a la educación de las mujeres se dieron, fue Paulina Vega. Oriunda de Boyacá, se formó en la Escuela Normal de Institutoras de Tunja y llegó a ser subdirectora de la Escuela Normal de Señoritas de Santa Marta. En 1932 fue seleccionada para recibir una beca y realizar estudios en Estados Unidos, en la Universidad Johns Hopkins, donde recibió el grado de Maestría en Ciencias (Cohen, 2001, p. 142-144). A su regreso y tras incidentes en la dirección del Colegio La Merced - primera institución que, según Cohen (2001), en Colombia ofreció formación a las mujeres en 1832-, fue nombrada como su directora y realizó allí gestiones que convirtieron a esta institución en la primera que en Colombia podía otorgar el título de bachilleres a las mujeres. Vega quiso continuar estudios de doctorado y solicitó apoyo económico a la fundación Rockefeller, pero fue rechazada por sus intensas actividades políticas en favor de las mujeres (Cohen, p. 19-21, 138-142, 306).

\section{Movilización femenina: búsqueda de participación}

Según lo plantea Cohen, en esas primeras décadas del siglo XX, hubo manifestaciones de las mujeres que querían participar de la educación secundaria que recibían los hombres. Uno de los eventos concretos fue el Cuarto Congreso Internacional Femenino, que en 1930 reunió a las mujeres que reclamaban mejores condiciones educativas y laborales, buscando además, cambios en el Código Civil que indicaba la forma en que debía desarrollarse la vida de las mujeres casadas. Con este tipo de iniciativas, las mujeres esperaban ingresar a la universidad, por un lado, porque la educación de ellas se centraba en cursos que no cumplían con los requisitos necesarios para que se formaran como bachilleres, y por el otro, porque el énfasis estaba en la emancipación de la mujer a través de la educación (Cohen, 1987, p. 307). El Congreso, que si bien no resultó importante por lo que allí ocurrió, sí permitió que las mujeres se identificaran con una lucha común y por ende, siguieron posteriormente manifestándose. 
En este congreso se plantearon problemas educativos que era necesario resolver. Al respecto - aunque de acuerdo con David Bushnell (2004), durante el gobierno de Olaya Herrera no hubo mayores reformas-, uno de los logros notables, fue

Un decreto que garantizaba a los colegios femeninos el derecho a conferir grados de bachiller, requisito indispensable para el ingreso a la universidad. Como resultado, en 1938, alrededor de seis de los 284 estudiantes graduados de todas las universidades de Colombia fueron mujeres (...). (Bushnell, 2004, p. 254)

Asimismo, una de las iniciativas que durante este gobierno se presentaron, se relaciona con el nombramiento de la primera mujer en el Ministerio de Educación Nacional, la señora Rosenda Torres, quien era oriunda de Antioquia y había iniciado su formación como maestra en el Colegio de María, en Yarumal; posteriormente, fue contratada en el naciente Gimnasio Moderno, y más adelante, fue subdirectora del Instituto Pedagógico Nacional para Señoritas, el cual representó un escenario clave en las transformaciones que se alcanzaron en cuanto a la educación femenina (Cohen, 2001, p. 127-128). Como lo señala Cohen, resultó importante su labor en el Ministerio, puesto que incluso en el diario El Tiempo se resaltó, aunque fuera en forma caricaturesca, su labor ${ }^{2}$.

Tanto Paulina Gómez Vega como Rosenda Torres tuvieron la posibilidad de formarse como maestras en ciudades distintas a Bogotá. Como lo señaló Helg (1987), accedieron a la alternativa que las mujeres de clase media tenían para educarse y que como lo propuso Radke (1936), era la mejor opción para las hijas de dicha clase dadas las escasas profesiones femeninas, con lo cual no solo se buscaba solucionar el asunto de su necesidad de ocupación, sino la necesidad de terminar con la "odiosa costumbre de entregar a los niños pequeños al cuidado de una sirvienta ignorante" (p. 21).

\section{Interdependencias y condiciones de acceso a la educación}

En este punto, es necesario reconocer que si las primeras mujeres lograron acceder a la educación, fue en gran parte porque "la mayoría de ellas había crecido en familias cuyos padres tenían el firme convencimiento de que la educación es fuente de progreso, tanto para sus hijas como para sus hijos (...)" (Cohen, 2001, p. xiii). En la Revista 
Hogar se publicó una entrevista en la que una maestra de escuela, la señora Margot Gnecco de Cuellar, hija de un magistrado de la Corte Suprema de Justicia, fue formada en labores prácticas como la mecanografía y la contabilidad, y señalaba la importancia de la formación para sus hijos:

(...) la más grande satisfacción de mi vida, será el día en que vea a mi Nena formada de manera que ella pueda valerse por sí sola. Son tres mis chiquitines, a quienes ya quisiera empezar a instruir, pues lo único positivo es lo que se aprende. En Bogotá todavía no se acostumbran a que la mujer de posición distinguida trabaje, y miran con cierto desdén a las empleadas; pero poco a poco irá implantándose la idea de que es conveniente aprender a valerse por sí mismas (...). El día en que las madres nos preocupemos más por enseñar a nuestras hijas el valor del carácter, la aritmética y la química, cambiará un poco la simple situación de la mujer actual. (Camacho, 1926c, p. 5)

Ahora bien, es cierto que el interés en este texto se centra en lo que las mujeres maestras hicieron para que el acceso femenino a la educación superior se ampliara; sin embargo, es pertinente señalar cómo otras mujeres desde otros ángulos de la vida pública hicieron su parte en esa empresa. Al respecto, es destacada la actividad de promoción que desde la Revista Hogar, mencionada previamente, hizo Ilva Camacho, la primera mujer periodista en el país. Desde el inicio de la revista en 1926 y pese a que tuvo apenas dos años de circulación, Ilva Camacho se pronunció de manera reiterada respecto a la necesidad de garantizar la instrucción de las mujeres. Bajo el pseudónimo de "Campesina", escribió varias notas en las que motivaba a sus lectoras a usar las letras en su defensa. En “iCuántas cosas diría si supiera escribir!”, uno de sus artículos, señaló el anhelo más grande, que según ella, estaba en la mujer:

Jamás he deseado cambiarme por mujer alguna, bella, rica o poderosa; pero quisiera ser esa mujer que puede desahogar su alma escribiendo y va dejando su palabra hecha monumentos de arte y como esculpida en el espíritu de los lectores, sugiriéndoles la esencia de lo grandioso, de lo bello, de lo verdadero, como un eco o un reflejo de lo que se ha sentido al vertir (sic) el espíritu en palabras. Cuando pienso en esto, reconozco que la instrucción es la primera necesidad de la mujer. (Camacho, 1926b, p. 4)

En otro de sus documentos escribía a sus lectoras con ahínco: 
De la manera más solemne y formal encarecemos a quienes lean estas líneas, detenerse un instante a meditar sobre la enorme conveniencia que para la evolución femenina significa, en los actuales momentos, este acontecimiento trascendental [el acceso a la educación]; y si no es la elegida, procure participar la idea a sus amigas; de esta manera surgirá sin duda, la preciosa vanguardia que espera el porvenir de nuestro sexo para decidir sus destinos (...). Juzgamos suficientemente inteligentes a las lectoras de esta revista para comprender que ya está descartada la idea de que una doctora queda exceptuada de los encantos y ternuras concernientes a la mujer de hogar; éstos, como el perfume de las flores, permanecerán intactos en ellas, realzándose con los atributos de la ciencia. (Camacho, 1926d, p. 3)

En la revista, Ilva Camacho presentaba textos suyos y de otros autores, a través de los cuales mezclaba hábilmente la promoción de las costumbres de la época respecto a la higiene y el mantenimiento del hogar, en el que la mujer tenía un papel importante ${ }^{3}$, con la provocación liberal y crítica respecto al papel tradicional de la mujer en la escritura:

Sin haber escrito nunca más que cartas de familia, es emocionante el momento de empezar a escribir algo que otros ojos menos cariñosos y naturalmente más severos han de ver y de someter a la crítica.

Con todo, va llegando la hora de sacudir el mutismo que nos han impuesto los prejuicios del medio y de nuestra propia timidez. ¿Por qué hemos de conformarnos con ser las eternas, las silenciosas, las débiles excluidas del mundo del espíritu? Si por deficiencias intelectuales o de educación no tenemos una ilustración suficiente para alagar a los eruditos y exquisitos [,] que nos lean al menos [,] que se nos permita exhibir en humildes pero sinceras palabras algo de lo mucho que sabemos sentir y de lo mucho más que podemos hacer. (Camacho, 1926a, p. 14)

A principios del veinte, época en la que se forman Paulina Vega y Rosenda Torres, la opción de formación femenina se encontraba casi de manera exclusiva en las escuelas normales. Dichas instituciones iniciaron labores en Colombia desde el siglo XIX (Báez, 2002, p. 157-180) y formaban a las mujeres, inicialmente, solo para ser docentes de

\footnotetext{
Ver por ejemplo Sofía Ospina de Navarro, "Nuestra cultura”, Revista Hogar, 73, junio 27 de 1927. En este texto señala, respecto a las críticas que el feminismo y la educación de las mujeres despertaba, que "el verdadero feminismo no hace bachilleras; forma mujeres conscientes, prontas a acometer con entusiasmo las buenas obras sociales; y esposas abnegadas que saben ser consuelo de sus maridos y que tienen comprensión suficiente para oírles hablar con interés de sus proyectos y negocios" (p. 5).
} 
educación primaria (Cohen, 2001, p. 9). Esta temprana incursión femenina en el escenario educativo tuvo consecuencias importantes para las siguientes generaciones.

Así, por ejemplo, se reconoce que como resultado de este acceso, hubo mujeres de clase alta que participaron en la creación de colegios y de empresas comerciales, los cuales abrieron un importante espacio laboral para otras mujeres. En este sentido, el proceso de industrialización, de acuerdo con Renán Vega, implicó una modernización sin modernidad, dado que, según él, el desarrollo capitalista no estuvo acompañado de cambios culturales de fondo (Vega, 2002, p. 13). Contrario a esto, Herrera (1995) expone que el capitalismo sí trajo al país cambios culturales significativos y que además, generó interdependencias políticas y económicas con otras naciones. En el marco de estos cambios, Colombia se consolidó como exportador de café y garantizó con ello un vínculo estable en el mercado internacional. Según esta autora, estas transformaciones en la economía permitieron el fortalecimiento de la industria y la inversión en infraestructura (Herrera, 1995).

Con dichas transformaciones económicas, en la nación se dio entonces una incorporación importante de la mujer en el campo laboral, dado que hubo una creciente demanda de mano de obra, lo que generó un amplio escenario en las empresas productoras de cigarrillos, cerveza y chocolate, las cuales fueron posteriormente reemplazadas por la industria textil (Martínez, 1997, p. 29). Según Bushnell (2004):

La mayoría de la fuerza laboral estaba constituida por mujeres jóvenes de poblaciones cercanas o provenientes de familias de la clase obrera local, quienes ganaban treinta centavos de dólar por día. Además, recibían de sus patronos un tratamiento especial que incluía dormitorios cuidadosamente vigilados para las muchachas que venían de poblaciones lejanas y por lo tanto estaban separadas de sus familias, capillas para que no descuidaran las obligaciones religiosas y cursos para la superación personal durante las horas libres. (p. 238)

En este contexto, se ofrecía a principios de la década del 20 educación femenina que, además de ofrecerles formación como maestras, les ofrecía formación en asuntos comerciales, lo cual les permitió a las mujeres ocuparse como secretarias o emplearse en oficinas públicas (Martínez, 1997, p. 29). No obstante, para entonces, como lo señala Martínez (1997), ya se gestaban reclamaciones por parte de las mujeres, dadas las diferencias salariales, las condiciones de higiene y los abusos sexuales de los cuales eran objeto en muchos casos (Martínez, 1997, p. 29). 
Asimismo, era clara la inconformidad respecto a la educación diferenciada entre hombres y mujeres, la cual les daba a los primeros la oportunidad de realizar estudios universitarios. Solo será hasta 1933 cuando se alcance la igualdad respecto a los planes de estudio ofrecidos para hombres y mujeres, lo que les permitirá a ellas ingresar a la universidad (Decreto 227 de febrero 2 de 1933, Diario Oficial, 22215).

Mientras esto ocurría en el país, en otras latitudes se presentaban luchas para el reconocimiento de los derechos civiles y políticos de las mujeres, cuya influencia fue recibida aquí tanto por hombres como mujeres que se sentían insatisfechos respecto a la formación femenina ${ }^{4}$. Así por ejemplo, desde el Órgano de Facultad de Educación de la Universidad Nacional, se señalaba en 1933 no solo la necesaria igualdad entre hombres y mujeres, sino el atraso que en este asunto el país presentaba respecto a otras naciones. En primer lugar, se afirmó: "si se acepta que la mujer tenga derechos iguales a los del hombre, se debe admitir, como condición indispensable, la necesidad y el derecho que la mujer tiene para que se le instruya de igual manera que al hombre". En segundo lugar, se hizo énfasis en la manera en que otros países trabajaban por la igualdad entre hombres y mujeres: "hay veinte estados en el mundo en donde se ha reconocido el sufragio a la mujer. En esos estados ocupa puesto en el gobierno, en las cámaras legislativas, en la diplomacia, pero para llegar a ellos ha tenido previa preparación (...)" (Huertas, 1933, p. 44). Estas ideas liberales se encontraron entonces con valores que, de acuerdo con Herrera, estaban bastante arraigados en el país, pero con los cuales tuvieron que coexistir (Herrera, 1995).

Por su parte, Ilva Camacho también acudió a la experiencia de otras naciones respecto a la educación de la mujer, para mostrar dicha necesidad en el país. En este sentido, publicó entrevistas de extranjeras que en sus naciones habían recibido formación superior y que por diversas razones visitaban Colombia ${ }^{5}$. A esta periodista se le acusaba de feminista; por un lado, debido a los constantes señalamientos frente a la desigualdad entre hombres y mujeres en sus columnas, y por el otro, por las caricaturas que aparecían en la revista y en las que los hombres se mostraban supeditados a una mujer más fuerte que ellos ${ }^{6}$.

Ver por ejemplo Clara Luna, "Una campaña por el mejoramiento de la mujer”. Revista Hogar, 65, mayo 2 de 1927, p. 12.

Ver por ejemplo, Ilva Camacho, "Entrevistas femeninas de hogar. Con la Dra. Lydia F. de Grutzendler" Revista Hogar, 55, febrero 20 de 1927, p. 5. Ver también, Ilva Camacho, "Entrevistas femeninas de hogar. Con la Dra. Mary William". Revista Hogar, 55, febrero 27 de 1927, p. 5.

Ver anexo 2. 


\section{Alcances de las primeras luchas}

La instrucción recibida por las primeras generaciones de maestras permitió entonces que muchas otras no tuvieran ya que formarse exclusivamente como maestras, sino que contaran con opciones distintas. Empero, el Estado continuó por mucho tiempo haciendo condición necesaria la formación como docente para las mujeres que querían entrar a la universidad. En este sentido, señala Radke (1936), era necesario que las señoritas que aspiraran a obtener un certificado de segunda enseñanza que les permitiera su ingreso a las facultades universitarias, hicieran estudios en el Instituto Pedagógico Nacional para Señoritas o en las Escuelas Normales que tuvieran a su disposición en los departamentos (p. 17). En consecuencia, la formación docente se convertía en paso obligado para las mujeres, aun cuando aspiraran a formarse en carreras distintas.

Ahora bien, aunque en el Instituto Pedagógico para Señoritas se aceptaban las disposiciones respecto al paso de las mujeres por la formación docente, al mismo tiempo se hacían los esfuerzos necesarios para que las mujeres llegaran a la universidad. Así, desde el Instituto, y atendiendo a la normatividad que regía su creación, se solicitó la conformación de la Facultad de Ciencias de la Educación para Mujeres, en la Universidad Nacional, ya que el primer artículo que decretó la creación de dicho instituto, señalaba que las estudiantes debían tener formación en didáctica (Ley 25 de noviembre 6 de 1927, Diario Oficial 16239) y como lo señalaba Radke, no había en su institución condiciones para cumplir dicho mandato (Radke, 1936). En respuesta a esta solicitud, a través del decreto 857 de 1934, se creó entonces la Facultad de Ciencias de la Educación en la Universidad Nacional (Decreto 857 de abril 21 de 1934, Diario Oficial 22587). Esta creación tuvo consecuencias importantes, dado que, en primer lugar, permitió que los títulos que recibieran quienes se graduaran de esta facultad, tuvieran la misma categoría que los expedidos por la Universidad Nacional, es decir, títulos universitarios. En segundo lugar, decretó que a quienes vinieran formándose en el Instituto Pedagógico Nacional para Señoritas y que por tanto habían "perfeccionado sus estudios en las ciencias fisicoquímicas y biológicas" (Decreto 857 de abril 21 de 1934 Diario Oficial 22587), el Ministerio de Educación Nacional les expediría el título de licenciadas en dichas ciencias y además de ello, podrían aspirar al grado de doctoras en sus áreas de especialización. Gracias a estas disposiciones, para 1934 había ya cuatro mujeres que luego de formarse en el Instituto Pedagógico Nacional, obtuvieron el título de licenciadas en la Universidad Nacional y dos de ellas obtuvieron posteriormente, becas en el exterior: Magdalena Briceño y Josefina Rodríguez (Radke, 1936). 


\section{Conclusión}

Hasta aquí se evidencia que no es posible señalar una causa concreta a la cual adjudicarle el acceso de la mujer a la educación superior. Contrario a esto, se encuentran diversas variables que contribuyeron para que las mujeres en las primeras décadas del siglo XX tuvieran la posibilidad de formarse, en primera instancia, como maestras y luego, como universitarias. Para empezar, se halla la importante tarea de quienes se formaron como maestras. Aquellas pioneras que tuvieron en sus manos la formación de otras mujeres que más adelante podrían decidirse por caminos profesionales distintos. Además de ello, tuvieron influencia las dinámicas económicas que ampliaron el campo laboral y permitieron la ocupación femenina, que si bien se dio en condiciones de desigualdad respecto a los hombres, fue fundamental para que las mujeres se organizaran y reconocieran la necesidad de reclamar frente a ello. De otra parte, fue claro el influjo que sobre el pensamiento de hombres y mujeres tuvieron las experiencias que en otros países se daban, lo que generó expresiones públicas de inconformidad que tuvieron eco en la población. En este sentido, la prensa representó un espacio importante que divulgó, por un lado, lo que en otros países ocurría en relación con la formación de las mujeres, y por el otro, un sentimiento de inconformidad y reclamación. Dicha divulgación tuvo acogida en la medida en que se dio en un ambiente en el que no se rechazaban de manera categórica las buenas costumbres que debían permanecer en la mujer, sino que, usando su mismo lenguaje, aparentemente conservador, llegó a manos de las mujeres que reconocieron en las publicaciones de la Revista Hogar, por ejemplo, las transformaciones que debían darse para alcanzar condiciones un tanto más igualitarias respecto a los hombres. Finalmente, no hay que olvidar que, el acceso de las primeras mujeres a la formación de las consideradas profesiones liberales, "es un testimonio de los logros de sus familias, de las directoras de colegios y de las mujeres mismas que unieron sus recursos y movilizaron redes de apoyo que les permitieron alcanzar los objetivos de educación deseados" (Cohen, 2001, p. 309). 


\section{Referencias}

Báez Osorio, M. (2002). El surgimiento de las escuelas normales femeninas en Colombia. Revista Historia de la Educación Latinoamericana, 4, 157-180.

Bushnell, D. (2004). Colombia, una nación a pesar de sí misma. Tercera Edición. Bogotá: Editorial Planeta.

Camacho, I. (octubre 3 de 1926a). La página de nuestras lectoras. Revista Hogar, 36, p. 14.

Camacho, I. (noviembre 21 de 1926b). ¡Cuántas cosas diría si supiera escribir!. Revista Hogar, 43, p. 4.

Camacho, I. (noviembre 28 de 1926c). Entrevistas femeninas de «Hogar» con Doña Margot Gnecco de Cuellar. Revista Hogar, 44, p. 5.

Camacho, I. (diciembre 5 de 1926d). La mujer en la universidad. Revista Hogar, 45.

Camacho, I. (febrero 20 de 1927). Ilva Camacho, "Entrevistas femeninas de hogar. Con la Dra. Lydia F. de Grutzendler. Revista Hogar, 55.

Cohen, L. (2001). Colombianas en la vanguardia. Medellín: Editorial Universidad de Antioquia.

Helg, A. (1987). La educación en Colombia 1918-1957. Una historia social, económica y política. Bogotá: Fondo Editorial CEREC.

Herrera, M.C. (1995). Las mujeres en la historia de la educación. En M. Velásquez Toro (ed.), Las mujeres en la historia de Colombia, Tomo III. Bogotá: Editorial Norma.

Huertas, M. (1933). El feminismo en la educación. Educación. Órgano de la Facultad de Educación de la Universidad Nacional, I (1), 41- 46.

León, A. y Rojas D.M. (2005). Mujeres e historia. Centro de Investigaciones y Desarrollo Científico. Ciencias Sociales, 269-293.

Luna, C. (mayo 2 de 1927). Una campaña por el mejoramiento de la mujer. Revista Hogar, 65, p. 12.

Martínez, A. (1997). Presencia femenina en la historia de Colombia. Bogotá: Academia Colombiana de Historia XLIII.

Ospina de Navarro, S. (junio 5 de 1927). Nuestra cultura. Revista Hogar, 73, p. 5.

Radke, F. (1936). Historia del Instituto Pedagógico para Señoritas 1927-1935. Bogotá: Editorial El Gráfico.

Rendón, R. (julio 9 de 1931). "Cómo pasaron las cosas" El Tiempo.

Revista Hogar. (Octubre 14 de 1926), p. 9

Vega, R. (2002). Gente muy rebelde. Tomo I. Bogotá: Ediciones Pensamiento Crítico. 


\section{Anexos}

1.

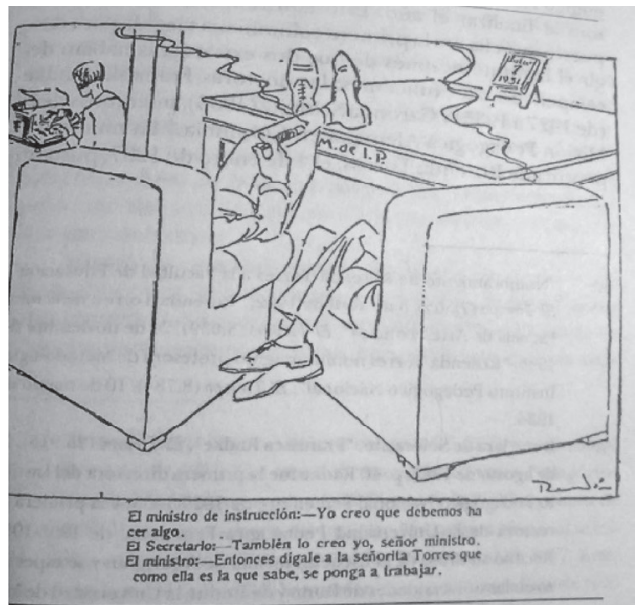

Cómo pasaron las cosas. (Rendón, 1931, citado por Cohen, 2001, p. 129)

2.

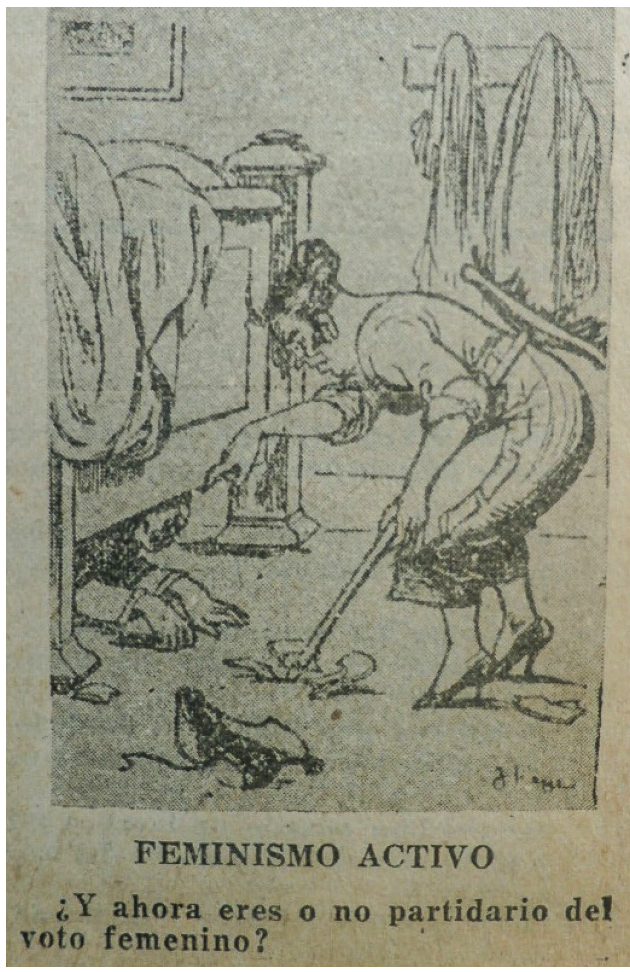

Feminismo activo. (Revista Hogar, octubre 14 de 1926, p. 9) 